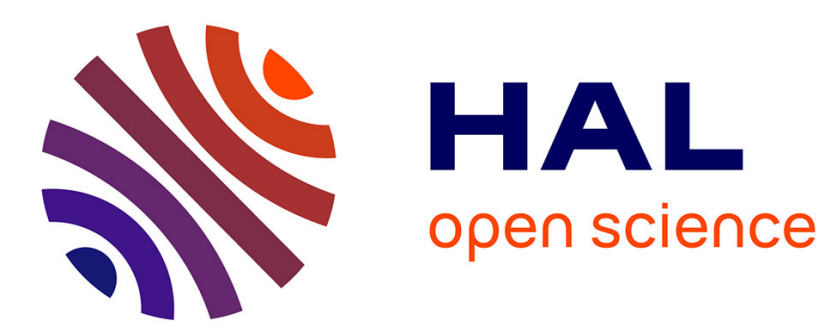

\title{
Experimental Evidence for a Liquid-Liquid Crossover in Deeply Cooled Confined Water
}

\author{
Antonio Cupane, Margarita Fomina, Irina Piazza, Judith Peters, Giorgio \\ Schirò
}

\section{- To cite this version:}

Antonio Cupane, Margarita Fomina, Irina Piazza, Judith Peters, Giorgio Schirò. Experimental Evidence for a Liquid-Liquid Crossover in Deeply Cooled Confined Water. Physical Review Letters, 2014, 113 (21), pp.215701. 10.1103/PhysRevLett.113.215701 . hal-01582604

\section{HAL Id: hal-01582604 \\ https://hal.science/hal-01582604}

Submitted on 6 Sep 2017

HAL is a multi-disciplinary open access archive for the deposit and dissemination of scientific research documents, whether they are published or not. The documents may come from teaching and research institutions in France or abroad, or from public or private research centers.
L'archive ouverte pluridisciplinaire HAL, est destinée au dépôt et à la diffusion de documents scientifiques de niveau recherche, publiés ou non, émanant des établissements d'enseignement et de recherche français ou étrangers, des laboratoires publics ou privés. 


\title{
Experimental Evidence for a Liquid-Liquid Crossover in Deeply Cooled Confined Water
}

\author{
Antonio Cupane, Margarita Fomina, and Irina Piazza \\ University of Palermo, Department of Physics and Chemistry, via Archirafi 36, 90123 Palermo, Italy \\ Judith Peters \\ Institut Laue Langevin, 71 avenue des Martyrs, F-38000 Grenoble, France, \\ Université Joseph Fourier, F-38041 Grenoble Cedex 9, France, \\ CNRS, CEA-Institut de Biologie Structurale, 71 avenue des Martyrs, 38000 Grenoble, France
}

Giorgio Schirò

CNRS-Institut de Biologie Structurale, 71 avenue des Martyrs, 38000 Grenoble, France

(Received 16 June 2014; published 21 November 2014)

\begin{abstract}
In this work we investigate, by means of elastic neutron scattering, the pressure dependence of mean square displacements (MSD) of hydrogen atoms of deeply cooled water confined in the pores of a three-dimensional disordered $\mathrm{SiO}_{2}$ xerogel; experiments have been performed at 250 and $210 \mathrm{~K}$ from atmospheric pressure to 1200 bar. The "pressure anomaly" of supercooled water (i.e., a mean square displacement increase with increasing pressure) is observed in our sample at both temperatures; however, contrary to previous simulation results and to the experimental trend observed in bulk water, the pressure effect is smaller at lower $(210 \mathrm{~K})$ than at higher $(250 \mathrm{~K})$ temperature. Elastic neutron scattering results are complemented by differential scanning calorimetry data that put in evidence, besides the glass transition at about $170 \mathrm{~K}$, a first-order-like endothermic transition occurring at about $230 \mathrm{~K}$ that, in view of the neutron scattering results, can be attributed to a liquid-liquid crossover. Our results give experimental evidence for the presence, in deeply cooled confined water, of a crossover occurring at about $230 \mathrm{~K}$ (at ambient pressure) from a liquid phase predominant at $210 \mathrm{~K}$ to another liquid phase predominant at $250 \mathrm{~K}$; therefore, they are fully consistent with the liquid-liquid transition hypothesis.
\end{abstract}

DOI: 10.1103/PhysRevLett.113.215701

PACS numbers: 64.70.Ja, 64.70.pm, 25.40.Dn

Liquid water is a ubiquitous and fascinating physical system. Its peculiar behavior (the so-called water anomalies, like, e.g., the temperature dependence of isobaric specific heat or the pressure dependence of isothermal transport coefficients, etc.), already present at temperatures above $0{ }^{\circ} \mathrm{C}$ and becoming evident in the supercooled regime, has challenged physicists for a long time and has served as a test for theories and hypotheses on soft condensed matter. Despite many years of active research and different proposed scenarios [1-4], a commonly accepted explanation for the anomalous water behavior has not yet been reached. Here we focus on the so-called "pressure anomaly," i.e., on the anomalous increase of the isothermal diffusion coefficients observed for liquid water at low temperatures (below $\sim 300 \mathrm{~K}$ ) and getting more and more evident in the supercooled region. Note that, in the same temperature range, the isobaric temperature dependence of transport properties also exhibits an anomalous behavior consistent with a power-law form: $X(T) \propto$ $\left(T / T_{s}-1\right)^{\gamma}$ [5]; this implies that approaching a critical temperature $T_{s}$ (usually around $220 \mathrm{~K}$, at atmospheric pressure) transport properties, like, e.g., viscosity or the inverse diffusion coefficient, should diverge. The commonly accepted, qualitative explanation of the anomalous pressure effect is that pressure disrupts the tetrahedral hydrogen bond network with a consequent increase of molecular mobility; in contrast, compression of a "normal" liquid leads to a progressive loss of mobility as the molecules are brought closer to each other [6]. The pressure dependence of translational and rotational diffusion coefficients of bulk water has been reported in Refs. [6-9]. Results are expressed as the pressure dependence of the ratio $R(P)=X(P) / X\left(P_{\mathrm{atm}}\right)$, where $X$ stands for the measured transport property. Water behaves as a normal liquid (i.e., $R$ decreases with pressure) at temperatures above the melting point, while the anomalous behavior (i.e., $R$ increases with pressure) is present already at $273 \mathrm{~K}$ and gets strongly enhanced when water is supercooled to $243 \mathrm{~K}$, where $R(P=1 \mathrm{kbar})$ is between 1.6 and 2 . The pressure effect on translational diffusion is smaller than that on rotational diffusion and exhibits a maximum located at about 1.5-2.0 kbar for translation and about 2.5-3.0 kbar for rotation. Unfortunately, due to homogeneous nucleation, experiments on bulk water at temperatures below $243 \mathrm{~K}$ are impossible; however, given the divergence of the inverse diffusion coefficient at $T_{s}$, the anomalous pressure effect is expected to increase on lowering the temperature towards $T_{s}$. The qualitative explanation of these effects originates from the open network structure of water enhanced in the supercooled state. Simulation studies on 
the pressure dependence of the water translational diffusion coefficient using the extended simple point charge (SPC/E) potential have also been performed $[10,11]$ and allow for extending the temperature range investigated down to $210 \mathrm{~K}$. Simulations qualitatively reproduce the experimental data; it is very interesting to note that the pressure effect at $210 \mathrm{~K}$ is predicted to be much larger than at $240 \mathrm{~K}$, in line with the fact that the translational diffusion coefficient of SPC/E water tends to 0 at $T_{s} \sim 190 \mathrm{~K}$.

The aim of our experiments was to investigate the pressure dependence of the translational diffusion coefficient of deeply cooled confined water and to obtain experimental evidence of the liquid-liquid transition (LLT) from a higher temperature high-density liquid (HDL) to a lower temperature low-density liquid (LDL), originally suggested by Poole et al. [12] and recently proposed to occur at about $230 \mathrm{~K}$ (at ambient pressure) in deeply cooled water confined in MCM-41 [13-15], vycor [16], and in the hydration water of proteins [17], but also seriously questioned by computational and experimental studies [18-20]. On the other hand, a number of recent experimental and simulation studies suggested the existence of LLT in many atomic [21-23] and molecular [24,25] liquids; in this second case, LLT was suggested to be a quite general phenomenon arising from anisotropic interactions like, e.g., hydrogen bonding [25]. Turning back to water, the recent proposal that links the onset of the biologically relevant protein dynamics (the so-called protein dynamical transition) to a LLT in the protein hydration water [26-29] has added interest to the search of experimental evidence proving or disproving its real existence.

In our samples water was confined in the pores of a hydrophilic 3D-disordered silica matrix obtained with the sol-gel method through hydrolysis and polycondensation of the alcoxide precursor tetramethylortosilicate. Silica xerogels were prepared as already reported [30]. During the aging process the sample hydration $h=\operatorname{gr}\left[\mathrm{H}_{2} \mathrm{O}\right] / g r\left[\mathrm{SiO}_{2}\right]$ was monitored by weighting; when the $h$ value of 0.42 was reached, the xerogels were crunched to obtain hydrated powders; with the used procedure the 3D-disordered, porous, silica matrix has a broad distribution of pore sizes with average dimensions of about $20 \AA$ [31]. At the hydration $h=0.42$ the contribution of incoherent scattering from $\mathrm{H}$ nuclei accounts for $91 \%$ of the total scattering. We can then safely assume that the dynamic structure factor measured in our experiment is essentially related to the self-correlation function of hydrogen atoms. Our idea is simple: by means of elastic neutron scattering (ENS) we measure the pressure dependence of water hydrogen mean square displacement (MSD) up to $1.2 \mathrm{kbar}$ and at two temperatures: $250 \mathrm{~K}$ (above the putative LDL $\rightarrow$ HDL transition; water essentially in the HDL state) and $210 \mathrm{~K}$ (below the putative LDL $\rightarrow$ HDL transition [13]; water essentially in the LDL state). ENS experiments are complemented by Differential Scanning Calorimetry (DSC) experiments on the same samples, to characterize their thermodynamic state and to evidence the presence of calorimetric phase transitions.

For ENS experiments we used the high hydrostatic pressure equipment developed at the Institut Laue Langevin (ILL) in Grenoble (France) [32] for neutron scattering studies of powder and solution samples. The cylindrical cell, built of the high-tensile aluminium alloy (7049-T6), is $4 \mathrm{~mm}$ thick and can withstand pressure loads up to $1.5 \mathrm{kbar}$. To transmit the pressure homogeneously, we used Fluorinert ${ }^{\mathrm{TM}}$ liquid [33] that has a pour point of $178 \mathrm{~K}$ and was tested to be completely inert. This avoids using gas for pressure transmission and therefore possible artifacts arising from gas diffusion inside the matrix pores; on the other hand, Fluorinert ${ }^{\mathrm{TM}}$ diffusion was excluded by weighting the sample before and after the measurements. The stick was put inside the closed cycle dry cryostat of the backscattering spectrometer IN13 [34] at the ILL and cooled down to 210 or $250 \mathrm{~K}$. When the temperature was reached, the compressor of the cryostat was stopped, so as to avoid the cold point and thus freezing of the liquid transmitting the pressure. The temperature was controlled to stay constant along the data collection time. We measured each pressure and temperature point for $5-8 \mathrm{~h}$, at 210 and $250 \mathrm{~K}$, for pressure values between 20 and 1200 bar in steps of 300 bar. MSD values were obtained from elastic spectra with the usual procedure: $\mathrm{MSD}=-6 d \ln [I(Q, \omega=0)] / d Q^{2}$ [where $I(Q, \omega=0)$ is the scattering intensity at the elastic line, defined by the width of the resolution function $\Delta \omega=8 \mu \mathrm{eV}$ FWHM], in the limit of the Gaussian approximation when $Q \rightarrow 0$ [35]. On the other hand, limiting the analysis to $Q \leq 1.1 \AA^{-1}$ allows us to neglect contributions arising from rotational motions [13], so that, in the diffusion limit, MSD is related to the translational diffusion coefficient $D$ by the Einstein relation MSD $=6 D \tau_{\text {res }}$, where $\tau_{\text {res }}=100 \mathrm{ps,}$ for IN13. Calorimetric measurements were performed using a Diamond DSC Perkin-Elmer calorimeter with a cryofill device using liquid nitrogen as a cold source. Indium was used as a standard to calibrate temperature and heat flow. The heat flow error was $0.05 \mathrm{~mW}$. Samples were sealed in steel pans of $\sim 60 \mu \mathrm{l}$ and were first cooled to $123 \mathrm{~K}$ with $5 \mathrm{~K} / \mathrm{min}$ cooling rate, approximately the same cooling rate used in ENS experiments. After equilibration at $123 \mathrm{~K}$, calorimetric up-scans from 123 to $303 \mathrm{~K}$ were performed with $20 \mathrm{~K} / \mathrm{min}$ heating rate. An empty sealed pan was used as a reference. The baseline was measured at the same scan rate with no pans in the furnace. Three samples were measured, at hydration levels $h=0.05$ (i.e., a dry sample containing structural water not removable under vacuum), $h=0.19$ (where most of the water is "interfacial water," i.e., forming single water layers in direct interaction with the pores walls), and $h=0.42$ (identical to that used for ENS measurements); the thermograms measured at $h=0.05$ were used to subtract the matrix contribution.

Information on the thermodynamic state of the system has been obtained with DSC. The temperature dependence 


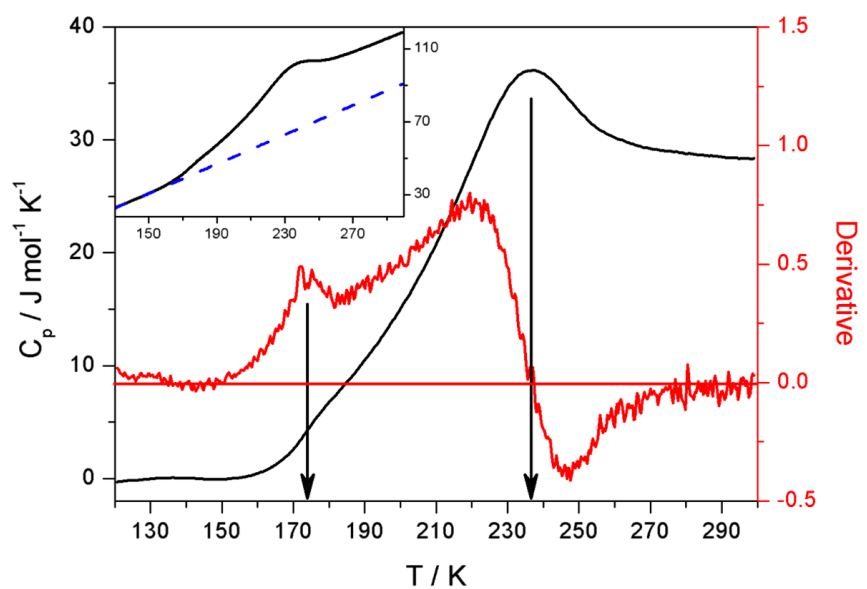

FIG. 1 (color online). Inset: Temperature dependence of molar specific heat $\left(C_{P}\right)$ of water confined in a silica hydrogel at $h=0.42$. The dashed line is a linear fit to the low-temperature behavior. Main: Temperature dependence of $C_{P}$ (black line) and its derivative (red line) after subtraction of the linear extrapolation. The vertical lines indicate the midpoint temperatures of the glass transition and of the specific heat maximum (liquid-liquid transition). Note the close similarity of our $C_{P}$ data with the calculations of Ref. [40].

of the molar specific heat $\left(C_{P}\right)$ is reported in the inset of Fig. 1; it compares very favorably with analogous data of Oguni et al. [36] [see Supplemental Material (SM) [37], Fig. S1] on water confined in MCM-41. As is clearly shown in the main panel of Fig. 1, our confined water exhibits a glass transition (step in $C_{P}$; peak in the derivative) at $T \sim 170 \mathrm{~K}$, followed by a first-order-like transition (maximum in $C_{P}$; positive-zero-negative sequence in the derivative) at $T \sim 230 \mathrm{~K}$; no signs of crystallization (minimum in $C_{P}$; negative-zero-positive sequence in the derivative) are observed in between. These experiments suggest the following picture: at low temperature, water confined within the pores of the silica matrix is in a glassy state; around $170 \mathrm{~K}$ it undergoes a glass $\rightarrow$ liquid transition followed by a first-order-like liquid $\rightarrow$ liquid transition at $\sim 230 \mathrm{~K}$. An analogous DSC experiment on the sample at $h=0.19$ (see SM [37], Fig. S2) evidenced the presence of a barely detectable broad glass transition at lower temperature with respect to $h=0.42$ (in agreement with a decrease of glass transition temperature when water strongly interacts with pore walls [36,38]), but there was no sign of the first-order-like transition. DSC results provided the following essential information: (1) at ambient pressure and at the temperatures investigated by ENS, water inside the xerogel is in a deeply cooled liquid state; (2) these temperatures correspond to liquid water below $(210 \mathrm{~K})$ and above $(250 \mathrm{~K})$ a liquid $\rightarrow$ liquid transition; (3) what is observed in the sample at $h=0.42$ at $\sim 230 \mathrm{~K}$ is a transition of the so-called "internal" water, not directly interacting with pore walls [39].

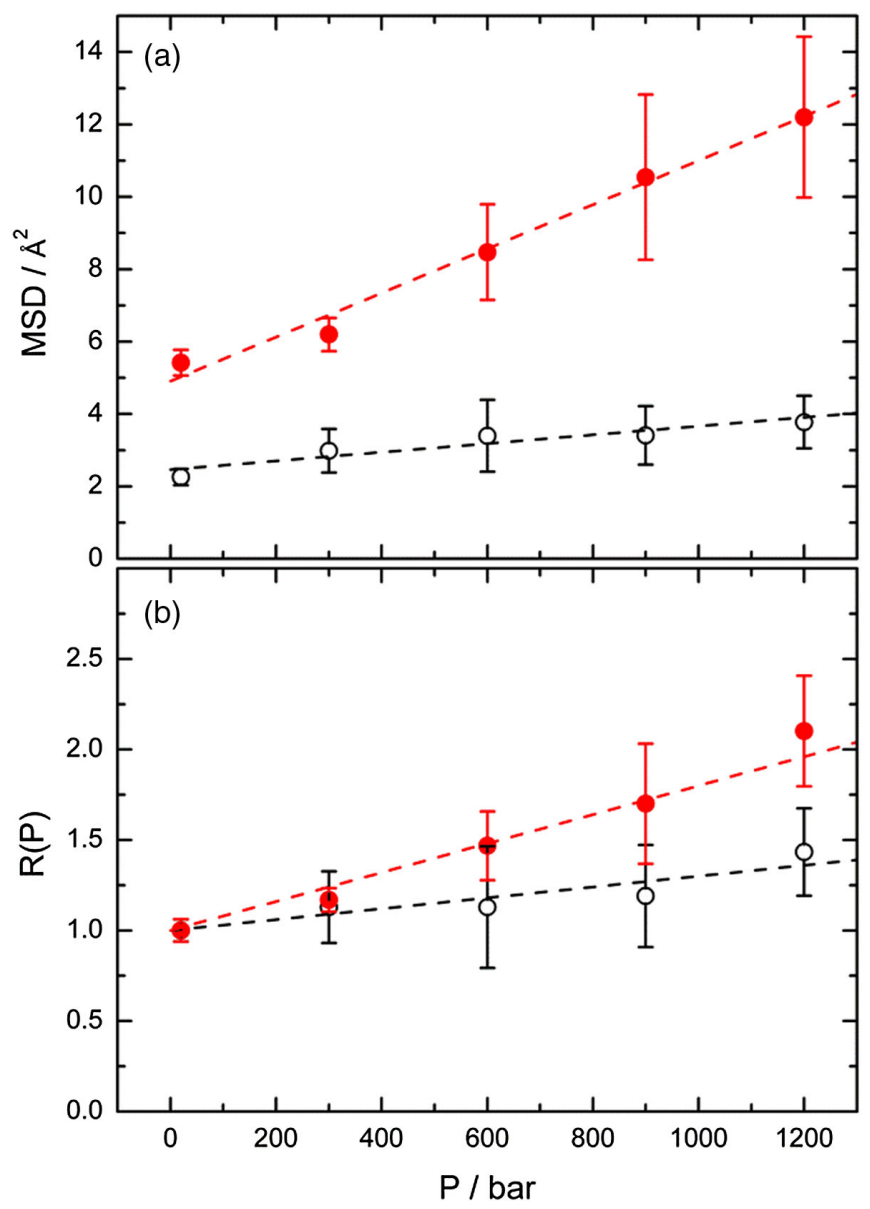

FIG. 2 (color online). (a) MSD and (b) $R(P)=$ $\operatorname{MSD}(P) / \operatorname{MSD}(P=20$ bar $)$ as a function of pressure. Black open circles, $T=210 \mathrm{~K}$; red circles, $T=250 \mathrm{~K}$. Dashed lines are linear fits.

The central result of the ENS experiment is shown in Fig. 2, where we report the MSD [Fig. 2(a)] and the quantity $R(P)=\operatorname{MSD}(P) / \operatorname{MSD}(P=20$ bar $)$ [Fig. 2(b)] as a function of pressure in the range 20-1200 bar at $T=210$ and $250 \mathrm{~K}$. More detailed data, i.e., normalized values of $\ln [I(Q, \omega=0)]$ versus $Q^{2}$ at the two temperatures investigated, are reported in the SM [37], Fig. S3. Results in Fig. 2 immediately reveal that the water hydrogen's MSD increases with pressure; i.e., the water pressure anomaly is observed in our sample, both at 250 and at $210 \mathrm{~K}$. However, the pressure effect is larger at $250 \mathrm{~K}$ than at $210 \mathrm{~K}$. The values of $d R(P) / d P$ obtained from the linear fits reported in Fig. 2 are 1.8 and $1.25 \mathrm{kbar}^{-1}$ at 250 and $210 \mathrm{~K}$, respectively. Unfortunately, the pressure range investigated does not allow us to put in evidence the possible presence and location of the $R(P)$ maxima. We also note that from the MSD measured at 20 bar (see Fig. S2 of the SM [37]) and using the relation $D=\mathrm{MSD} / 6 \tau_{\text {res }}$, one obtains $D(250 \mathrm{~K}, 20 \mathrm{bar})=1 \times 10^{-6} \mathrm{~cm}^{2} \mathrm{~s}^{-1}$ and $D(210 \mathrm{~K}, 20 \mathrm{bar})=$ $0.3 \times 10^{-6} \mathrm{~cm}^{2} \mathrm{~s}^{-1}$. These values compare favorably with $D$ values reported in the literature from quasielastic neutron 
scattering experiments or from simulations [11,41-43]. ENS results in Fig. 2 provide the dynamic counterpart of the thermodynamic description obtained by DSC (Fig. 1). First, the observation of an "anomalous" pressure effect on the MSD [together with the absence of Bragg diffraction from ice in the structure factor $I(Q)]$ confirms that in our sample water confined within the pores of the silica matrix is a liquid both at 250 and at $210 \mathrm{~K}$. Moreover, the behavior at $210 \mathrm{~K}$ is surprising: instead of an increased anomalous pressure effect, expected for a liquid approaching a critical divergence and indeed obtained for simulated SPC/E water [10,11], we measure a much lower effect. The glass transition or fragility scenario also seems unable to explain the reported effects. In fact, the "ideal" glass transition temperature $\left(T_{0}\right)$ of water has been reported to increase slightly with pressure, at about $5 \mathrm{~K}$ for a pressure increase of $1.5 \mathrm{kbar}[7,44]$; this, in turn, would cause a (rather small) increase of the quantity $T_{0} / T$ and therefore a viscosity increase, contrary to what was observed. Therefore, our data indicate that, going from 250 to $210 \mathrm{~K}$, water confined in the silica xerogel undergoes a crossover in which the hydrogen bond network becomes stronger and less deformable by pressure. This idea is then compatible with the existence of two different phases of water at 250 and $210 \mathrm{~K}$, i.e., with the LLT hypothesis. The pressure dependence of MSD is also compatible with the fragile-to-strong dynamic crossover in the water relaxation times observed by neutron scattering [13], nuclear magnetic resonance [14], and dielectric spectroscopy [28] in the same temperature region of the LLT. Indeed, relaxation times of fragile systems are more affected by pressure than those of strong systems. Supercooled water, in particular, shows a 1 order of magnitude reduction of translational relaxation times in the fragile regime when pressure is increased up to $1.4 \mathrm{kbar}$, while the pressure effect is much weaker in the strong regime $[13,45]$.

In conclusion, the pressure-dependent ENS data presented in this work shed light on the physical state of deeply cooled water confined in the 3D disordered $\mathrm{SiO}_{2}$ matrix and, together with calorimetric data, provide new experimental evidence of the presence of a crossover in the behavior of confined water occurring at about $230 \mathrm{~K}$. In fact, at $210 \mathrm{~K}$ water is in a liquid state, as shown by the fact that it exhibits an anomalous pressure effect (increasing MSD as pressure is increased) and confirmed by the thermodynamic transitions probed by DSC; however, the comparatively small pressure effect indicates the presence of an almost fully developed, locally icelike, hydrogen bond network less deformable by pressure and suggests that water is essentially in the LDL state. By increasing the temperature, a first-order-like liquid-liquid calorimetric transition is observed at $\sim 230 \mathrm{~K}$ so that at $250 \mathrm{~K}$ water is essentially in the HDL state in which the local, tetrahedrally coordinated, hydrogen bond network is not fully developed and is therefore more deformable by a pressure increase. Therefore, our data give experimental support to the LLT hypothesis.

The authors thank J. L. Barrat for fruitful discussions and the ILL for according the neutron beam time.

*antonio.cupane@unipa.it

[1] H. E. Stanley, P. Kumar, S. Han, M. G. Mazza, K. Stokeley, S. V. Buldyrev, G. Franzese, F. Mallamace, and L. Xu, J. Phys. Condens. Matter 21, 504105 (2009).

[2] F. Mallamace, C. Branca, M. Broccio, C. Corsaro, N. Ganzalez-Segredo, J. Spooren, H. E. Stanley, and S.-H. Chen, Eur. Phys. J. Spec. Top. 161, 19 (2008).

[3] C. E. Bertrand, Y. Zhang, and S.-H. Chen, Phys. Chem. Chem. Phys. 15, 721 (2013).

[4] M. G. Mazza, K. Stokeley, H. E. Stanley, and G. Franzese, J. Chem. Phys. 137, 204502 (2012).

[5] W. S. Price, H. Ide, Y. Arata, and O. Söderman, J. Phys. Chem. B 104, 5874 (2000).

[6] M. R. Arnold and H. D. Lüdemann, Phys. Chem. Chem. Phys. 4, 1581 (2002).

[7] E. W. Lang and H. D. Lüdemann, Ber. Bunsen-Ges. Phys. Chem. 85, 603 (1981).

[8] F. X. Prielmeier, E. W. Lang, R. J. Speedy, and H. D. Lüdemann, Phys. Rev. Lett. 59, 1128 (1987).

[9] F. X. Prielmeier, E. W. Lang, R. J. Speedy, and H. D. Lüdemann, Ber. Bunsen-Ges. Phys. Chem. 92, 1111 (1988).

[10] F. W. Starr, S. Harrington, F. Sciortino, and H. E. Stanley, Phys. Rev. Lett. 82, 3629 (1999).

[11] F. W. Starr, F. Sciortino, and H. E. Stanley, Phys. Rev. E 60, 6757 (1999).

[12] P. H. Poole, F. Sciortino, U. Essmann, and H. E. Stanley, Nature (London) 360, 324 (1992).

[13] L. Liu, S.-H. Chen, A. Faraone, C. W. Yen, and C. Y. Mou, Phys. Rev. Lett. 95, 117802 (2005).

[14] S.-H. Chen, F. Mallamace, C. Y. Mou, M. Broccio, C. Corsaro, A. Faraone, and L. Liu, Proc. Natl. Acad. Sci. U.S.A. 103, 12974 (2006).

[15] F. Mallamace, M. Broccio, C. Corsaro, A. Faraone, U. Wanderlingh, L. Liu, C. Y. Mou, and S.-H. Chen, J. Chem. Phys. 124, 161102 (2006).

[16] J.-M. Zanotti, M.-C. Belissent-Funel, and S.-H. Chen, Europhys. Lett. 71, 91 (2005).

[17] S.-H. Chen, L. Liu, E. Fratini, P. Baglioni, A. Faraone, and E. Mamontov, Proc. Natl. Acad. Sci. U.S.A. 103, 9012 (2006).

[18] D. T. Limmer and D. Chandler, J. Chem. Phys. 135, 134503 (2011).

[19] W. Doster, S. Busch, A. M. Gaspar, M. S. Appavou, J. Wuttke, and H. Scheer, Phys. Rev. Lett. 104, 098101 (2010).

[20] S. Pawlus, S. Khodadadi, and A. P. Sokolov, Phys. Rev. Lett. 100, 108103 (2008).

[21] Y. Katayama, T. Mizutani, W. Utsumi, O. Shimomura, M. Yamakata, and K. Funakoshi, Nature (London) 403, 170 (2000).

[22] C. Tien, E. V. Charnaya, W. Wang, Y. A. Kumzerov, and D. Michel, Phys. Rev. B 74, 024116 (2006).

[23] S. Sastry and C. A. Angell, Nat. Mater. 2, 739 (2003).

[24] R. Kurita and H. Tanaka, Science 306, 845 (2004). 
[25] R. Kurita and H. Tanaka, J. Phys. Condens. Matter 17, L293 (2005).

[26] P. Kumar, Z. Yan, L. Xu, M. G. Mazza, S. V. Buldyrev, S.-H. Chen, S. Sastry, and H. E. Stanley, Phys. Rev. Lett. 97, 177802 (2006).

[27] G. Schirò, F. Natali, and A. Cupane, Phys. Rev. Lett. 109, 128102 (2012).

[28] G. Schirò, M. Fomina, and A. Cupane, J. Chem. Phys. 139, 121102 (2013).

[29] M. Fomina, G. Schirò, and A. Cupane, Biophys. Chem. 185, 25 (2014).

[30] M. D'Amico, G. Schirò, A. Cupane, L. D'Alfonso, M. Leone, V. Militello, and V. Vetri, Langmuir 29, 10238 (2013).

[31] M. Cammarata, M. Levantino, A. Cupane, A. Longo, A. Martorana, and F. Bruni, Eur. Phys. J. E 12, 63 (2003).

[32] J. Peters, M. Trapp, D. Hughes et al., High Press. Res. 32, 97 (2012).

[33] V. A. Sidorov and R. A. Sadykov, J. Phys. Condens. Matter 17, S3005 (2005).

[34] F. Natali, J. Peters, D. Russo et al., Neutron News 19, 14 (2008).
[35] M. Tehei, D. Madern, C. Pfister, and G. Zaccai, Proc. Natl. Acad. Sci. U.S.A. 98, 14356 (2001).

[36] M. Oguni, Y. Kanke, A. Nagoe, and S. Namba, J. Phys. Chem. B 115, 14023 (2011).

[37] See Supplemental Material at http://link.aps.org/ supplemental/10.1103/PhysRevLett.113.215701 for supplemental figures.

[38] M. Oguni, S. Maruyama, K. Wakabayashi, and A. Nagoe, Chem. Asian J. 2, 514 (2007).

[39] A. Nagoe, Y. Kanke, M. Oguni, and S. Namba, J. Phys. Chem. B 114, 13940 (2010).

[40] D. M. Murphy and T. Koop, Q. J. R. Meteorol. Soc. 131, 1539 (2005).

[41] D. Paschek and A. Geiger, J. Phys. Chem. B 103, 4139 (1999).

[42] J. Qvist, H. Schober, and B. Halle, J. Chem. Phys. 134, 144508 (2011).

[43] J. Qvist, C. Mattea, E. P. Sunde, and B. Halle, J. Chem. Phys. 136, 204505 (2012).

[44] H. D. Lüdemann and E. W. Lang, J. Phys. (Paris), Colloq. 45, C7-41 (1984).

[45] S.-H. Chen, F. Mallamace, L. Liu et al., AIP Conf. Proc. 982, 39 (2008). 